\title{
High-sensitivity biosensor for simultaneous detection of cancer and diabetes using photonic crystal microstructure
}

Mojtaba Hosseinzadeh Sani

Imam Reza University

Ashkan Ghanbari

Islamic Azad University Central Tehran Branch

Hamed Saghaei ( $\nabla$ h.saghaei@iaushk.ac.ir)

Islamic Azad University Shahrekord Branch https://orcid.org/0000-0001-5588-3726

\section{Research Article}

Keywords: Photonic crystal biosensor, high sensitivity, blood cancer, diabetes, figure of merit

Posted Date: April 27th, 2021

DOI: https://doi.org/10.21203/rs.3.rs-451630/v1

License: (1) This work is licensed under a Creative Commons Attribution 4.0 International License.

Read Full License

Version of Record: A version of this preprint was published at Optical and Quantum Electronics on November 24th, 2021. See the published version at https://doi.org/10.1007/s11082-021-03371-3. 


\title{
High-sensitivity biosensor for simultaneous detection of cancer and diabetes using photonic crystal microstructure
}

\author{
Mojtaba Hosseinzadeh Sani ${ }^{1}$, Ashkan Ghanbaril ${ }^{2}$, and Hamed Saghael ${ }^{3,}$, \\ ${ }^{1}$ Department of Electrical Engineering, Imam Reza International University, Mashhad, Iran \\ ${ }^{2}$ Department of Electrical Engineering, Central Tehran Branch, Islamic Azad University, Tehran, Iran \\ ${ }^{3}$ Department of Electrical Engineering, Shahrekord Branch, Islamic Azad University, Shahrekord, Iran \\ *Corresponding Author: h.saghaei@iaushk.ac.ir
}

\begin{abstract}
In this study, we propose a refractive index sensor for the simultaneous detection of cancer and diabetes based on photonic crystals $(\mathrm{PhC})$. The proposed $\mathrm{PhC}$ composed of silicon rods in the air bed arranged in a hexagonal lattice forms the fundamental structure, and two tubes are used to place the cancerous or diabetic samples for measurement. The sensor's transmission characteristics are simulated and analyzed by solving Maxwell's electromagnetic equations using the finite-difference time-domain method for samples under study. The diagnosis of three types of cancer and diabetes is based on changing the samples' refractive index by applying the laser source centered at $1550 \mathrm{~nm}$. Our results demonstrate that the proposed structure's quality factor and sensitivity can be adjusted by changing the sensor's geometry. They reveal that the transmission power is between $91 \% \sim 100 \%$, depending on the sample. The sensitivity range is also between 1294 and $3080 \mathrm{~nm} / \mathrm{RIU}$. The maximum figure of merit is about $1550.11 \mathrm{RIU}^{-1}$ with a detection range of $31 \times 10^{-6} \mathrm{RIU}$. The small biosensor area of $61.56 \mu \mathrm{m}^{2}$ makes it a suitable device for various applications in compact photonic integrated circuits.
\end{abstract}

Keywords: Photonic crystal biosensor, high sensitivity, blood cancer, diabetes, figure of merit.

\section{Introduction}

Integrated optical circuits (IOCs) have significant advantages over electronic integrated circuits (EICs). IOCs have a much higher propagation speed, very low loss, low data transmission error, and very high security compared to electrons in EICs. In recent years, photonic crystals (PhCs) have played an important role in designing all-optical circuits due to their unique properties. One of these features is their photonic bandgap (PBG) that prevents lights of certain wavelengths from propagating in one, two, or any number of polarization directions within the PhC structure. PhCs are designed in one, two, and three-dimensional structures, whose two-dimensional structure has been considered by researchers due to its greater applications and fabrication simplicity. Research groups designed a variety of structures using two-dimensional PhCs such as optical filters [1-5], logic gates [6-8], encoders and decoders [9-12], comparators [13,14], multiplexers and demultiplexers [15,16], adders and subtractors [17-24], registers [25,26], memories [27-29], splitters [30-32], analog-to-digital converters [33-35], optical fibers [36-43], sensors [44-50], PhC fibers [38,42,51-58], switches [59-62], interferometers [63-65], as well as all-optical clocked sequential circuits including flip-flops [66,67], synchronous and asynchronous counters $[68,69]$. 
In recent years, the refractive index sensor based on Fano resonance has become an active research topic [70]. Fano resonance is formed by the interference between discrete-state energy levels (narrow non-radiative dark mode) and continuous-state energy bands (broad radiant, bright mode), and it results in an asymmetrical and sharp linear graph [71]. Therefore, they are widely used in optical switches, nonlinearity, slow-optical devices, and biosensors [72-74]. Liu et al. proposed a planar meta-material sensor with electromagnetic induction transparency with a sensitivity of $588 \mathrm{~nm} / \mathrm{RIU}$ [75]. Guo Yuan et al. studied a new type of symmetrical plasma structure composed of a metal-insulator-metal (MIM) waveguide and a semi-ring short tube to control the asymmetric linear shape resonance wavelength, and it had a sensitivity of $575 \mathrm{~nm} / \mathrm{RIU}$ [76]. Zhou Jinli et al. designed a sensor with a trapezoidal structure to study the refractive index's sensitivity by filling a half-wavelength waveguide and a refractive index medium with a sensitivity of approximately $750 \mathrm{~nm} / \mathrm{RIU}$ [77]. Zafar et al. proposed a double elliptical ring-shaped resonator that can be used to detect the concentration of hemoglobin in the blood, with a sensitivity of 1100 $\mathrm{nm} / \mathrm{RIU}$ [78]. Wang Mengmeng et al. developed a nanosensor with a baffle and a circular resonant cavity to achieve a double Fano resonance with a sensitivity of $1114.3 \mathrm{~nm} / \mathrm{RIU}$ [79].

There are two main methods used to identify biological cells. In the conventional method, labels are used to identify the characteristics of biological cells, which is a traditional and invasive method, and in the second method, non-invasive and unlabeled tools are used to diagnose cell conditions that there are no markers required to identify the analytes. Since no labels are attached to the molecules, their true information and nature remain intact. Biosensors can also be used in cancer research to analyze target cell lines or protein changes in the cell. All-optical biosensors are suitable structures for fast detection. The main function of PhC-based biosensors is based on changing the refractive indices of the samples. The structure designed in this work can simultaneously detect three types of cancer and diabetes which improves the structure's performance from existing sensors. On the other hand, it is possible to detect their disease by having samples of two people simultaneously. The proposed device can be used as an appropriate method to accelerate cancer diagnosis and diabetes in critical situations and crowded hospitals. The paper is organized as follows. The mathematical background and physical structure of the biosensor are presented in Section 2. In Section 3, the numerical results achieved by solving Maxwell's electromagnetic equations using the finite-difference time-domain (FDTD) method are discussed. The paper is closed by the conclusion in Section 4.

\section{Mathematical background and physical structure}

\subsection{Mathematical background}

In this study, the FDTD method is used to solve Maxwell's equations. The desired biosensor has meshed with tiny grids. The grid size $(\Delta \mathrm{x}, \Delta \mathrm{y})$ is selected with different values in the FDTD solution. We set the boundary condition of the waveguide as a perfectly matched layer to absorb the electromagnetic waves. The propagation of light in a $\mathrm{PhC}$ structure is obtained by solving Maxwell's electromagnetic equations as follows 


$$
\nabla \times\left(\frac{1}{\varepsilon} \nabla \times H\right)=\left(\frac{\omega}{c}\right)^{2} H
$$

where $\varepsilon$ is the permittivity and $\omega$ is the frequency. The quality factor is another key indicator used to measure the overall performance of the sensor. It is calculated as follows:

$$
Q . f=\frac{\lambda_{0}}{F W H M}
$$

where $\lambda_{0}$ is the output resonant wavelength, and $F W H M$ represents full width at half maximum (FWHM) of the optical signal related to the sensor's resolution. The following equation expresses the biosensor sensitivity

$$
S=\frac{\Delta \lambda_{0}}{\Delta n}\left(\frac{n m}{R I U}\right)
$$

Here $\Delta \lambda_{0}$ is the amount of change in the wavelength in Fano resonance and $\Delta n$ denotes the amount of change in the refractive index. The figure of merit (FOM) is positively correlated with the performance and capability of the sensor and can be expressed by the following formula

$$
F O M=\frac{S}{F W H M}
$$

To describe the waveguide structure's transmission characteristics more appropriately, we used the FDTD for quantitative analysis. The output power is defined as $P_{\text {out }}$, and the input power is $P_{\text {in, }}$ and the transmittance ratio is $T=P_{\text {out }} / P_{\text {in }}$.

\subsection{PhC biosensor}

The proposed structure is shown in Fig. 1. It has two linear tubes for cancer and diabetes cells' simultaneous diagnosis in one or two people. A PhC composed of silicon rods in the air bed arranged in a hexagonal lattice with a lattice constant of $a=600 \mathrm{~nm}$ forms the fundamental structure, and two tubes are used to place the samples for measurement. The radii of the red and cyan tubes are $\mathrm{R}_{\mathrm{C} 1}=0.9 \mathrm{a}$, and $\mathrm{R}_{\mathrm{C} 2}=1.1 \mathrm{a}$.

Using the plane wave expansion (PWE) method, the fundamental structure's photonic band diagram has been calculated. Our results demonstrates that there is a wide normalized band gap in TM polarization mode at $0.276<\mathrm{a} / \lambda<0.446$, which is equal to $1345 \mathrm{~nm}<\lambda<2173 \mathrm{~nm}$ for $\mathrm{a}=600$ $\mathrm{nm}$. This bandwidth covers $\mathrm{C}$ and $\mathrm{L}$ optical transmission bands. The lowest optical fiber loss is in the C-band (1530-1565 $\mathrm{nm}$ ) and is generally used in many transmission applications. The L-band $(1565-1625 \mathrm{~nm})$ is the second lowest-loss wavelength band and is a popular choice when using the C-band is not sufficient to meet the bandwidth demand. A sample of human blood is placed inside the red tube, and a cyan tube is filled with a sample of tear, and a tunable laser source centered at $\lambda=1550 \mathrm{~nm}$ is applied to the biosensor's input waveguide. The resonance wavelength shifts due to the samples' refractive indices, and two resonant wavelengths are received simultaneously at the device output. All the parameters used in designing the proposed structure are listed in Table 1. 


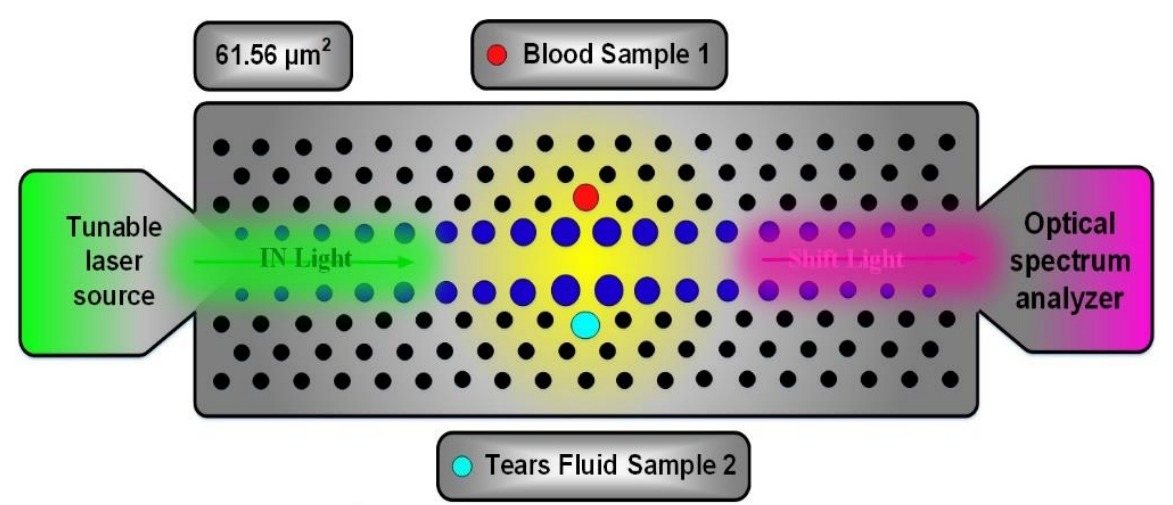

Fig. 1. The proposed structure of PhC-based biosensor.

Table 1. The PhC-based biosensor parameters.

\begin{tabular}{lccc}
\hline Parameter & Symbol & Quantity & Unit \\
\hline Cental wavelength & $\lambda_{0}$ & 1550 & $\mathrm{~nm}$ \\
Normalized band gap (TM) & $a / \lambda$ & $0.276-0.446$ & - \\
The corresponding photonic band gap & $\lambda$ & $1345-2173$ & $\mathrm{~nm}$ \\
Lattice constant & $\mathrm{a}$ & 600 & $\mathrm{~nm}$ \\
Radius of rods & $\mathrm{r}$ & 120 & $\mathrm{~nm}$ \\
Background refractive index (air) & $\mathrm{n}_{\mathrm{gb}}$ & 1 & - \\
Linear refractive index & $\mathrm{n}_{0}$ & 3.46 & - \\
The red tube radius & $\mathrm{R}_{\mathrm{C} 1}$ & 540 & $\mathrm{~nm}$ \\
The cyan tube radius & $\mathrm{R}_{\mathrm{C} 2}$ & 660 & $\mathrm{~nm}$ \\
X length & $\Delta \mathrm{x}$ & 5400 & $\mathrm{~nm}$ \\
Y length & $\Delta \mathrm{y}$ & 11400 & $\mathrm{~nm}$ \\
\hline
\end{tabular}

\section{Result and Discussion}

In Fig. 2a, blood and tear samples of healthy people with refractive indices of 1.36 and 1.35 are placed inside the red and cyan tubes, respectively. As shown in the figure, two resonance wavelengths are received at the output; these resonance wavelengths represent healthy people. The lightwave has been applied to the structure, and the shifted resonant wavelength for a healthy blood sample is equal to $\lambda_{\mathrm{s} 2}=1.593 \mu \mathrm{m}$, and also the shifted wavelength for a healthy tear sample is equal to $\lambda_{\mathrm{s} 1}=1.562 \mu \mathrm{m}$. If we get these two resonance wavelengths at the output of our structure, we will find that the person or people are healthy, and there are no cancer or diabetes cells in the samples. Important parameters in an optical biosensor are sensitivity, FOM, quality factor, FWHM, and detection limits (DL). As shown in Fig. 2, for the normal tear sample having a refractive index of 1.35 , a resonance is centered at $1.562 \mu \mathrm{m}$. It has the FWHM $=1.8 \mathrm{~nm}$ and FWHM is $2.2 \mathrm{~nm}$ for a normal blood sample with a refractive index of 1.36. In detecting a healthy person without cancer cells and diabetes cells, the sensitivity value is $S=3080 \mathrm{~nm} / \mathrm{RIU}$, and the figure of merit is $\mathrm{FOM}=1550.11 \pm 150.11 \mathrm{RIU}^{-1}$. The transmission spectra are $95 \%$ for sample \#1 
and $100 \%$ for sample \#2. Figure $2 \mathrm{~b}$ shows the resonance wavelengths of normal blood and tear samples in $\mathrm{dB}$ scale.
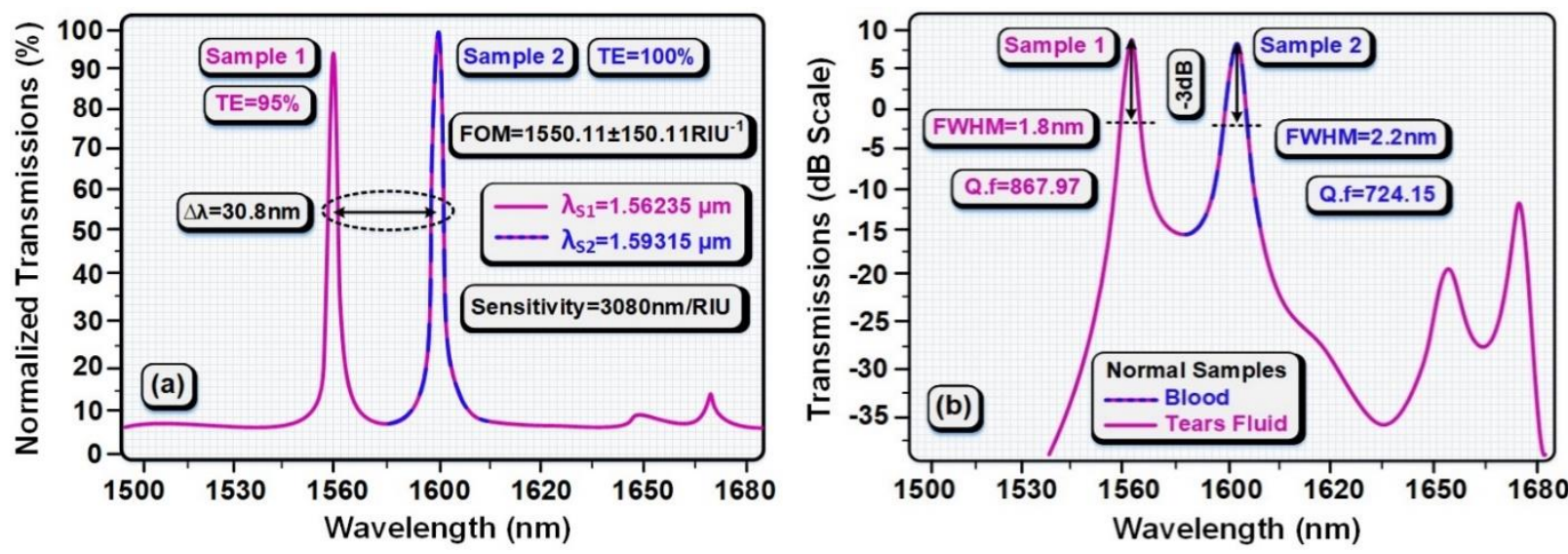

Fig. 2. Resonance wavelengths of normal blood and tear samples, (a) Normalized resonance power, (b) Normalized resonance power in $\mathrm{dB}$ scale.

Table 2 shows the refractive indices of the three types of normal and cancerous blood cells. It also represents the refractive indices of a healthy person and a diabetic person. According to these refractive indices, we study their resonant wavelength to identify the disease.

Table 2. Refractive indices of three types of normal and cancerous blood cells and normal and diabetic tear cells.

\begin{tabular}{lccc}
\hline Analytic Used & Refractive Index & Tears Sample \#2 & RI \\
\hline Blood Sample \#1 & RI & Normal Cells of diabetes & 1.350 \\
\hline Basal cell (Normal) & 1.360 & Effected Cells of diabetes & 1.410 \\
Basal cell (Cancerous) & 1.380 & & \\
HeLa cell (Normal) & 1.368 & & \\
HeLa Cell (Cancerous) & 1.392 & & \\
MDA-MB-231 Cell (Normal) & 1.385 & & \\
MDA-MB-231 Cell (Cancerous) & 1.399 & & \\
\hline
\end{tabular}

The normal basal cells are placed in the red tube (sample \#1) of the proposed biosensor shown in Fig. 1, and the basal cancer cells are placed in the cyan tube (sample \#2). Basal cancer cells are formed in the skin's outer layer (epidermis) due to intense sun exposure. These cells do not spread to other parts of the body. The cancer cells have a refractive index of 1.38, while the normal cells have a refractive index of 1.36. The sensor output spectrum has been plotted in Fig. 3a, where each case shows a specific resonance wavelength. The FWHM is about $2.2 \mathrm{~nm}$ for normal basal cells while it is $2 \mathrm{~nm}$ for basal cancer cells, and the FOM is $940.475 \pm 025 \mathrm{RIU}^{-1}$ with a sensitivity of S $=1893 \mathrm{~nm} / \mathrm{RIU}$. Figure $3 \mathrm{~b}$ shows the sensor output for detecting HeLa cancer cells. HeLa cells 
become cancerous due to infection with the human papillomavirus 18 (HPV18). The refractive index of these cells is 1.392, while the normal HeLa cell line has a refractive index of 1.368. The FWHM of the output signal is $2.2 \mathrm{~nm}$ for normal and FWHM $=1.9 \mathrm{~nm}$ for HeLa cell carcinoma, and the FOM $=940.475 \pm 025 \mathrm{RIU}^{-1}$ and sensitivity is $\mathrm{S}=1642 \mathrm{~nm} / \mathrm{RIU}$ and transmissions power for normal, and cancer HeLa cells are $\mathrm{TE}_{\mathrm{N}}=99 \%$ and $\mathrm{TE}_{\mathrm{H}}=95 \%$. MDA-MB-231 is extracted from the human chest and isolated from the pleural disease from a breast cancer patient [22]; the level of the FOM suitability shape value for FOM $=6097 \mathrm{RIU}^{-1}$ cancer cells. Figure $3 \mathrm{c}$ shows the resonance wavelengths for the MDA-MB-231 normal cell and MDA-MB-231 cancer cell.
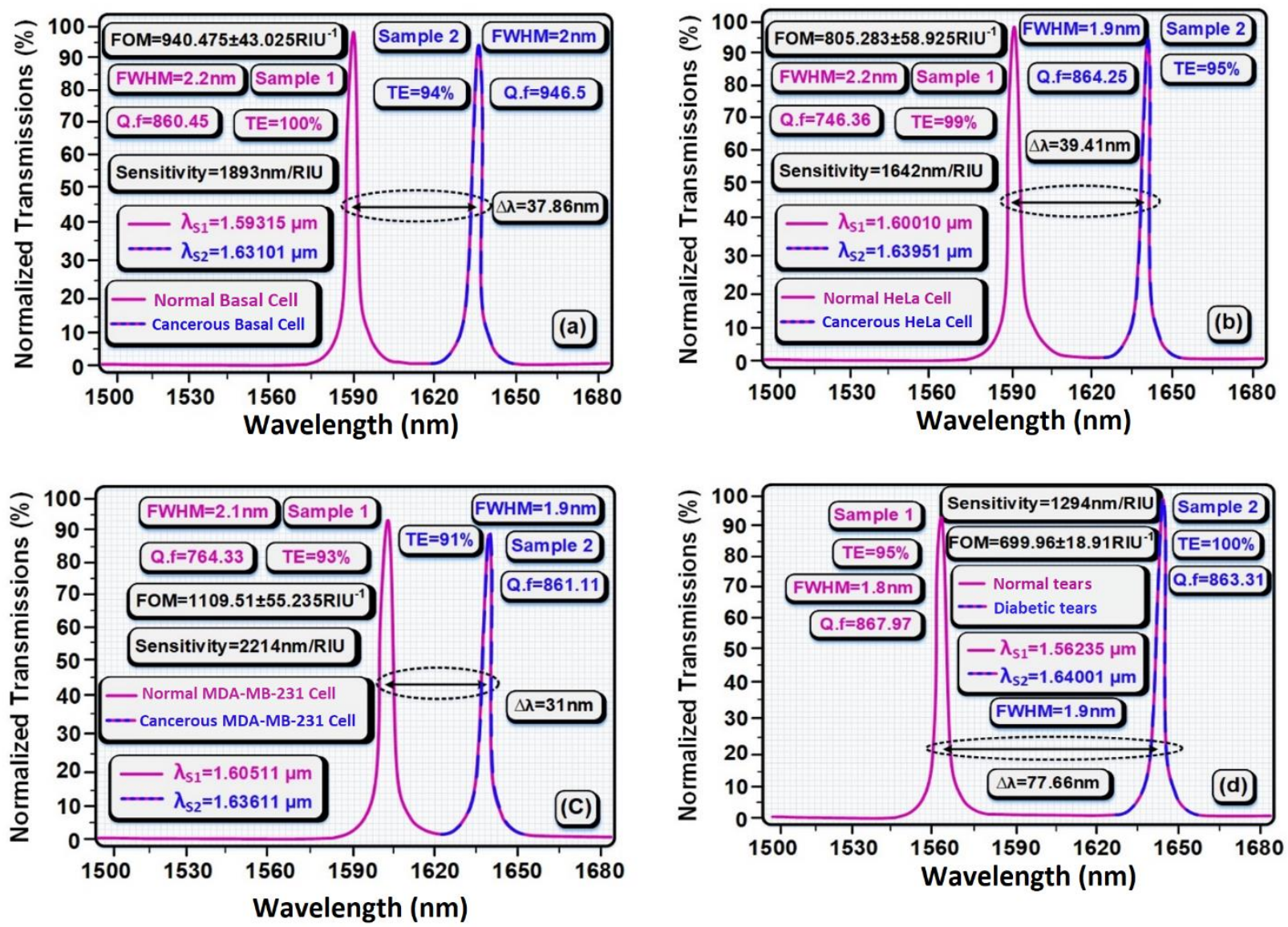

Fig. 3. Resonant wavelengths of normal and cancerous cells for (a) Basal cell, (b) HeLa cell, and (c) MDAMB-231 cell and also (d) normal and diabetic tears.

The refractive index of this type of cancer is 1.399 , and the normal cells of breast have a refractive index of 1.385. As mentioned in the previous sections, the tear sample is used to diagnose diabetes and normal cells have a refractive index of 1.35, while this coefficient for diabetics is about 1.41. Figure $3 \mathrm{~d}$ shows the resonance wavelength for normal and diabetic cells at the sensor output. It demonstrates that the sensor sensitivity is $\mathrm{S}=1294 \mathrm{~nm} / \mathrm{RIU}$, and transmissions powers for normal, and diabetic cell are $\mathrm{TE}_{\mathrm{N}}=95 \%$ and $\mathrm{TE}_{\mathrm{H}}=100 \%$, respectively. The $\mathrm{FWHM}=1.8 \mathrm{~nm}$ for normal and $\mathrm{FWHM}=1.9 \mathrm{~nm}$ for diabetic samples. In this section, the effects of physical parameters such as the radius of red and green tubes on the transmission power were studied. Figure 4a shows the effect of red tube radius on transmission power, resonant wavelength, and FWHM. 

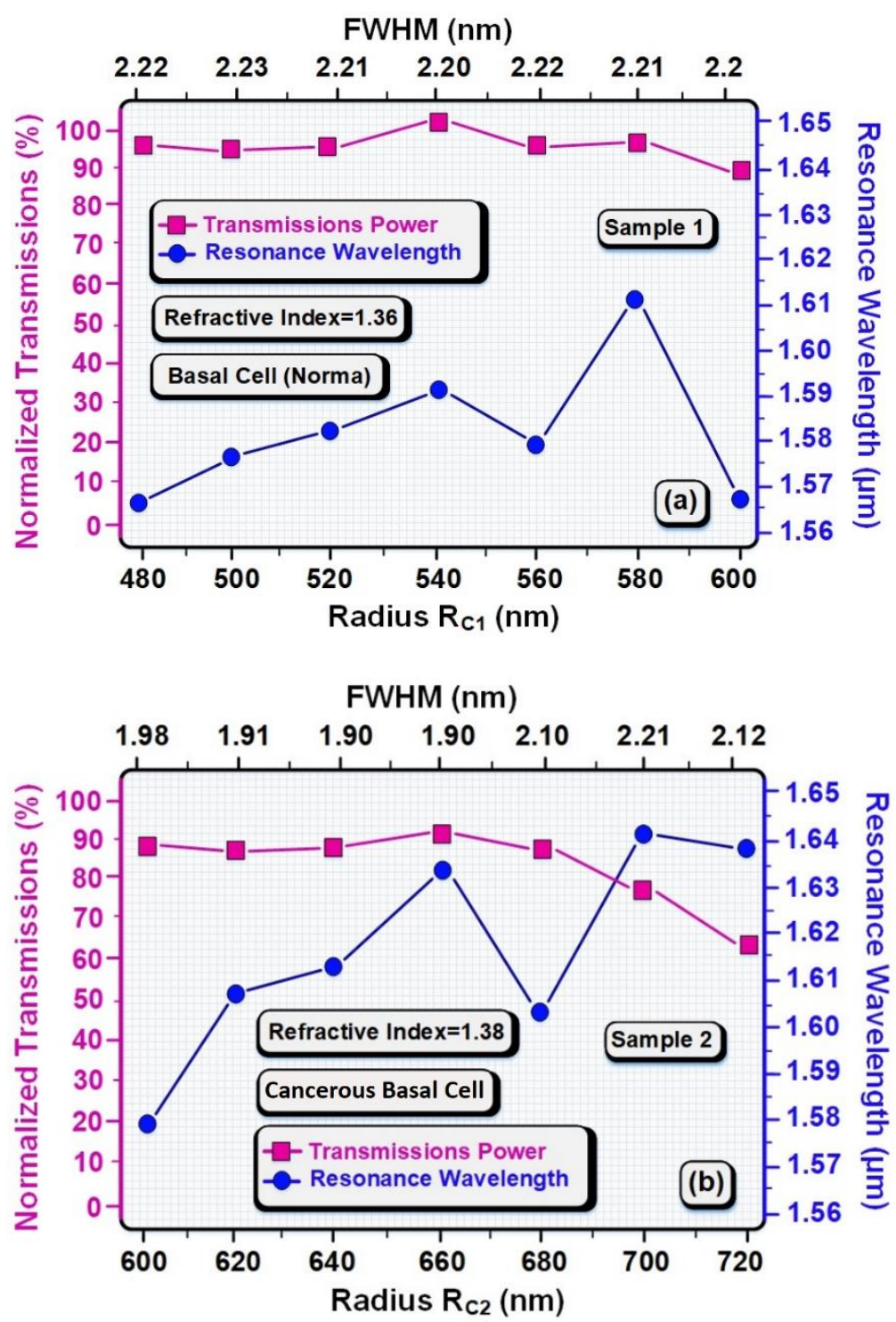

Fig. 4. The resonant wavelengths, transmission power, and FWHM for different tube radius. (a) Normal cell. (b) Cancer cell.

The results were calculated for the sample of normal basal cells with the refractive index of 1.36. As shown in this figure, the power transmission in a radius of $\mathrm{R}_{\mathrm{C} 1}=540 \mathrm{~nm}$ reaches $100 \%$. From this case, it can be concluded that the best radius to reduce power losses to zero and pass $100 \%$ of the transmitted power is this radius. On the other hand, the FWHM in different radii has different values. However, it has less value for $\mathrm{RC} 1=540 \mathrm{~nm}$, representing the lowest bandwidth's optical signal. Figure $4 \mathrm{~b}$ shows the effects of green tube radius with a refractive index of 1.38 on the basal cancer cell on important structural parameters. In this case, by increasing the radius to $\mathrm{R}_{\mathrm{C} 2}(<660)$ $\mathrm{nm}$, the amount of transmission power is almost constant, and by increasing the radius, the amount of transmission power decreases, and therefore in the radius of $\mathrm{R}_{\mathrm{C} 2}=660 \mathrm{~nm}$ with $94 \%$ transmission power is the best choice. The amount of bandwidth has also reached the lowest value in this radius. Therefore, we selected the best radii of $R_{C 1}=540 \mathrm{~nm}$ and $R_{C 2}=660 \mathrm{~nm}$ by reviewing 
and analyzing the results. Our results are shown in Table 3. In this table, the sensitivity parameter, quality factor, transmission power, average bandwidth, and figure of merit are calculated for different refractive indices for sample \#1 and sample \#2. The maximum sensitivity in the red tube is equal to $2214 \mathrm{~nm} / \mathrm{RIU}$ for MDA-MB-231 Cell (Cancerous). Table 4 also compares the essential parameters of the structure proposed in this paper with other proposed articles. As shown in the table, our proposed structure is better than other structures in terms of detection sensitivity, transmission power, quality factor, figure of merit, and simultaneous detection of two samples.

\section{Conclusion}

The designed sensor can detect a person with cancer and diabetes from a healthy person by a human blood sample and a tears sample. One feature of the proposed structure is the simultaneous detection of two samples using two tubes. Due to the importance of the accuracy and sensitivity parameter in the sensors' design, the FWHM is $1.8 \mathrm{~nm}$, and the FOM is about $1550.11 \mathrm{RIU}^{-1}$. The sensitivity is $3080 \mathrm{~nm} / \mathrm{RIU}$, and the resolution detection range is $31 \times 10^{-6} \mathrm{RIU}$.

Table 3. Calculation of important parameters using proposed biosensor with blood and tear samples.

\begin{tabular}{|c|c|c|c|c|c|c|c|}
\hline Analytic Used & $\mathbf{R I}$ & $\lambda_{0}(\mu \mathrm{m})$ & FWHM (nm) & Quality factor & T.E (\%) & FOM $\left(\right.$ RIU $\left.^{-1}\right)$ & $\mathbf{S}(\mathbf{n m} / \mathbf{R I U})$ \\
\hline \multicolumn{8}{|l|}{ Blood Sample \#1 } \\
\hline Basal cell (Normal) & 1.360 & 1.59315 & 2.2 & 840.45 & 100 & - & Ref \\
\hline Basal cell (Cancerous) & 1.380 & 1.63101 & 2.0 & 946.50 & 94 & 940.475 & 1893 \\
\hline HeLa cell (normal) & 1.368 & 1.60010 & 2.2 & 746.36 & 99 & - & Ref \\
\hline HeLa Cell (Cancerous) & 1.392 & 1.63951 & 1.9 & 864.25 & 95 & 805.283 & 1642 \\
\hline $\begin{array}{l}\text { MDA-MB-231 Cell } \\
\text { (normal) }\end{array}$ & 1.385 & 1.60511 & 2.1 & 464.33 & 93 & - & Ref \\
\hline $\begin{array}{l}\text { MDA-MB-231 Cell } \\
\text { (Cancerous) }\end{array}$ & 1.399 & 1.63611 & 1.9 & 861.11 & 91 & 1109.51 & 2214 \\
\hline \multicolumn{8}{|l|}{ Tears Sample \#2 } \\
\hline $\begin{array}{l}\text { Normal Cells of } \\
\text { diabetes }\end{array}$ & 1.350 & 1.56235 & 1.8 & 867.97 & 95 & - & Ref \\
\hline $\begin{array}{l}\text { Effected Cells of } \\
\text { diabetes }\end{array}$ & 1.410 & 1.64001 & 1.9 & 863.31 & 100 & 699.96 & 1294 \\
\hline
\end{tabular}


Table 4. Comparison of detection sample \#1 and sample \#2, quality factor, transmission power, and sensitivity parameters of the proposed sensor with the reported sensors.

\begin{tabular}{cccccc}
\hline Reference & $\begin{array}{c}\text { Detection } \\
\text { Sample }\end{array}$ & $\begin{array}{c}\text { Quality } \\
\text { factor }\end{array}$ & FOM (RIU-1) & $\begin{array}{c}\text { Transmission } \\
\text { power (\%) }\end{array}$ & $\begin{array}{c}\text { Sensitivity } \\
\text { (nm/RIU) }\end{array}$ \\
\hline Ref [20] & Blood & $650 \pm 50$ & $1400 \pm 200$ & 80 & 2500 \\
\hline Ref [21] & $\begin{array}{c}\text { Blood and } \\
\text { Tears }\end{array}$ & 1082 & - & - & 6.5764 \\
\hline Ref [22] & Glucose & - & - & 86 & 422 \\
\hline Ref [23] & Glucose & $1.11 \times 10^{5}$ & 1117 & 92 & 462 \\
\hline Ref [24] & Blood & 262 & - & 100 & - \\
\hline Ref [25] & - & - & 88 & 98 & 263 \\
\hline Ref [26] & - & 1264 & 84 & 90 & 840 \\
\hline This work & Blood, Tears & 946.50 & $1109.51 \pm 55.235$ & 100 & 3080 \\
\hline
\end{tabular}

\section{Funding statement}

This research received no specific grant from any funding agency in the public, commercial, or not-for-profit sectors.

\section{Conflict of Interest}

The authors declare that they have no conflict of interest.

\section{Reference}

1. Y. Guo, S. Zhang, J. Li, S. Li, en T. Cheng, "A sensor-compatible polarization filter based on photonic crystal fiber with dual-open-ring channel by surface plasmon resonance," Optik (Stuttg). 193, 162868 (2019).

2. H. Alipour-Banaei, M. Jahanara, en F. Mehdizadeh, "T-shaped channel drop filter based on photonic crystal ring resonator," Optik (Stuttg). 125(18), 5348-5351 (2014).

3. M. R. Rakhshani en M. A. Mansouri-Birjandi, "Realization of tunable optical filter by photonic crystal ring resonators," Optik (Stuttg). 124(22), 5377-5380 (2013).

4. S. Naghizade en H. Saghaei, "Tunable graphene-on-insulator band-stop filter at the mid-infrared region," Opt. Quantum Electron. 52(4), 224 (2020).

5. A. Foroughifar, H. Saghaei, en E. Veisi, "Design and analysis of a novel four-channel optical filter using ring resonators and line defects in photonic crystal microstructure," Opt. Quantum Electron. 53(2), (2021).

6. R. M. Younis, N. F. F. Areed, en S. S. A. Obayya, "Fully integrated and and or optical logic gates," IEEE Photonics Technol. Lett. 26(19), 1900-1903 (2014).

7. P. Andalib en N. Granpayeh, "All-optical ultracompact photonic crystal AND gate based on nonlinear ring resonators," J. Opt. Soc. Am. B 26(1), 10 (2009).

8. H. M. E. Hussein, T. A. Ali, en N. H. Rafat, "New designs of a complete set of Photonic Crystals logic gates," Opt. Commun. 411, 175-181 (2018).

9. S. Naghizade en H. Khoshsima, "Low input power an all optical $4 \times 2$ encoder based on triangular lattice shape photonic crystal," J. Opt. Commun. 1, 1-8 (2018).

10. T. A. Moniem, "All-optical digital $4 \times 2$ encoder based on 2D photonic crystal ring resonators," J. Mod. Opt. 63(8), 735-741 (2016).

11. S. Naghizade en H. Saghaei, "A novel design of all-optical 4 to 2 encoder with multiple defects in silica- 
based photonic crystal fiber," Optik (Stuttg). 222, 165419 (2020).

12. S. Naghizade, S. Mohammadi, en H. Khoshsima, "Design and simulation of an all optical 8 to 3 binary encoder based on optimized photonic crystal or gates," J. Opt. Commun. 410, 793-798 (2018).

13. V. Fakouri-Farid en A. Andalib, "Design and simulation of an all optical photonic crystal-based comparator," Optik (Stuttg). 172, 241-248 (2018).

14. H. Jile, "Realization of an all-optical comparator using beam interference inside photonic crystal waveguides," Appl. Opt. 59(12), 3714 (2020).

15. S. Naghizade en S. Mohammadi, "Optical four-channel demultiplexer based on air-bridge structure and graphite-type ring resonators," Photonic Netw. Commun. 40(1), 40-48 (2020).

16. S. Naghizade en S. M. Sattari-Esfahlan, "High-performance ultra-compact communication triplexer on silicon-on-insulator photonic crystal structure," Photonic Netw. Commun. 34(3), 445-450 (2017).

17. M. Hosseinzadeh Sani, A. Ghanbari, en H. Saghaei, "An ultra-narrowband all-optical filter based on the resonant cavities in rod-based photonic crystal microstructure," Opt. Quantum Electron. 52(6), 295 (2020).

18. R. Moradi, "All optical half subtractor using photonic crystal based nonlinear ring resonators," Opt. Quantum Electron. 51(4), 119 (2019).

19. H. Alipour-Banaei en H. Seif-Dargahi, "Photonic crystal based 1-bit full-adder optical circuit by using ring resonators in a nonlinear structure," Photonics Nanostructures-Fundamentals Appl. 24, 29-34 (2017).

20. M. J. Maleki, A. Mir, en M. Soroosh, "Design and analysis of a new compact all-optical full-adder based on photonic crystals," Optik (Stuttg). 227, 166107 (2021).

21. M. J. Maleki, A. Mir, en M. Soroosh, "Ultra-fast all-optical full-adder based on nonlinear photonic crystal resonant cavities," Photonic Netw. Commun. 41(1), 93-101 (2021).

22. M. R. Geraili, S. E. Hosseini, M. B. Tavakoli, en M. Shokooh-Saremi, "A proposal for an all optical full adder using nonlinear photonic crystal ring resonators," Optik (Stuttg). 199, 163359 (2019).

23. S. Naghizade en H. Saghaei, "A Novel Design of All-Optical Half-Adder Using a Linear Defect in Photonic Crystal Microstructure," J. Appl. Res. Electr. Eng. (2020).

24. S. Naghizade en H. Saghaei, "A novel design of all-optical full-adder using nonlinear X-shaped photonic crystal resonators," Opt. Quantum Electron. 53(3), 1-13 (2021).

25. N. Pahari en A. Guchhait, "All-optical Serial Data Transfer between Registers using optical non-linear materials," Optik (Stuttg). 123(5), 462-466 (2012).

26. M. Martinez-Dorantes, W. Alt, J. Gallego, S. Ghosh, L. Ratschbacher, Y. Völzke, en D. Meschede, "Fast Nondestructive Parallel Readout of Neutral Atom Registers in Optical Potentials," Phys. Rev. Lett. 119(18), 180503 (2017).

27. E. Kuramochi, K. Nozaki, A. Shinya, K. Takeda, T. Sato, S. Matsuo, H. Taniyama, H. Sumikura, en M. Notomi, "Large-scale integration of wavelength-addressable all-optical memories on a photonic crystal chip," Nat. Photonics 8(6), 474-481 (2014).

28. T. Alexoudi, G. T. Kanellos, en N. Pleros, "Optical RAM and integrated optical memories: a survey," Light Sci. Appl. 9(1), 1-16 (2020).

29. T. Uda, A. Ishii, en Y. K. Kato, "Single Carbon Nanotubes as Ultrasmall All-Optical Memories," ACS Photonics 5(2), 559-565 (2018).

30. S. Naghizade en S. Mohammadi, "Design and engineering of dispersion and loss in photonic crystal fiber 1 $\times 4$ power splitter (PCFPS) based on hole size alteration and optofluidic infiltration," Opt. Quantum Electron. 51(1), 17 (2019).

31. F. Parandin, M. R. Malmir, M. Naseri, en A. Zahedi, "Reconfigurable all-optical NOT, XOR, and NOR logic gates based on two dimensional photonic crystals," Superlattices Microstruct. 113, 737-744 (2018).

32. H. Saghaei, A. Zahedi, R. Karimzadeh, en F. Parandin, "Line defects on As2Se3-Chalcogenide photonic crystals for the design of all-optical power splitters and digital logic gates," Superlattices Microstruct. 110, 133-138 (2017).

33. S. Naghizade en H. Saghaei, "An ultra-fast optical analog-to-digital converter using nonlinear X-shaped photonic crystal ring resonators," Opt. Quantum Electron. 53(3), 1-16 (2021).

34. F. Mehdizadeh, M. Soroosh, H. Alipour-Banaei, en E. Farshidi, "All optical 2-bit analog to digital converter using photonic crystal based cavities," Opt. Quantum Electron. 49(1), 38 (2017).

35. A. Tavousi en M. A. Mansouri-Birjandi, "Optical-analog-to-digital conversion based on successive-like approximations in octagonal-shape photonic crystal ring resonators," Superlattices Microstruct. 114, 23-31 (2018).

36. A. Ghanbari, A. Kashaninia, A. Sadr, en H. Saghaei, "Supercontinuum generation with femtosecond optical pulse compression in silicon photonic crystal fibers at $2500 \mathrm{~nm}$," Opt. Quantum Electron. 50(11), (2018). 
37. H. Saghaei, V. Heidari, M. Ebnali-Heidari, en M. R. Yazdani, "A systematic study of linear and nonlinear properties of photonic crystal fibers," Optik (Stuttg). 127(24), 11938-11947 (2016).

38. H. Saghaei, M. Ebnali-Heidari, en M. K. Moravvej-Farshi, "Midinfrared supercontinuum generation via As_2Se_3 chalcogenide photonic crystal fibers," Appl. Opt. 54(8), 2072 (2015).

39. H. Saghaei, M. K. Moravvej-Farshi, M. Ebnali-Heidari, en M. N. Moghadasi, "Ultra-Wide Mid-Infrared Supercontinuum Generation in As40Se60 Chalcogenide Fibers: Solid Core PCF Versus SIF," IEEE J. Sel. Top. Quantum Electron. 22(2), (2016).

40. M. Aliee, M. H. Mozaffari, en H. Saghaei, "Dispersion-flattened photonic quasicrystal optofluidic fiber for telecom C band operation," Photonics Nanostructures - Fundam. Appl. 40, 100797 (2020).

41. A. Ghanbari, A. Kashaninia, A. Sadr, en H. Saghaei, "Supercontinuum generation for optical coherence tomography using magnesium fluoride photonic crystal fiber," Optik (Stuttg). 140, 545-554 (2017).

42. M. Diouf, A. Ben Salem, R. Cherif, H. Saghaei, en A. Wague, "Super-flat coherent supercontinuum source in As_388Se_612 chalcogenide photonic crystal fiber with all-normal dispersion engineering at a very low input energy," Appl. Opt. 56(2), 163 (2017).

43. H. Saghaei, "Supercontinuum source for dense wavelength division multiplexing in square photonic crystal fiber via fluidic infiltration approach," Radioengineering 26(1), 16-22 (2017).

44. F. Tavakoli, F. B. Zarrabi, en H. Saghaei, "Modeling and analysis of high-sensitivity refractive index sensors based on plasmonic absorbers with Fano response in the near-infrared spectral region," Appl. Opt. 58(20), (2019).

45. A. A. Tabrizi, H. Saghaei, M. A. Mehranpour, en M. Jahangiri, "Enhancement of absorption and effectiveness of a perovskite thin-film solar cell embedded with Gold nanospheres," Plasmonics (2021).

46. S. M. Alden Mostaan en H. Saghaei, "A tunable broadband graphene-based metamaterial absorber in the farinfrared region," Opt. Quantum Electron. 53(2), 96 (2021).

47. A. Kowsari en H. Saghaei, "Resonantly enhanced all-optical switching in microfibre Mach-Zehnder interferometers," Electron. Lett. 54(4), 229-231 (2018).

48. A. Farmani, M. Soroosh, M. H. Mozaffari, en T. Daghooghi, "Optical nanosensors for cancer and virus detections," in Nanosensors for Smart Cities (Elsevier, 2020), bll 419-432.

49. A. Farmani en A. Mir, "Graphene sensor based on surface plasmon resonance for optical scanning," IEEE Photonics Technol. Lett. 31(8), 643-646 (2019).

50. M. H. Sani en S. Khosroabadi, "A Novel Design and Analysis of High-Sensitivity Biosensor Based on Nano-Cavity for Detection of Blood Component, Diabetes, Cancer and Glucose Concentration," IEEE Sens. J. 20(13), 7161-7168 (2020).

51. H. Saghaei, "Dispersion-engineered microstructured optical fiber for mid-infrared supercontinuum generation," Appl. Opt. 57(20), 5591 (2018).

52. M. Ebnali-Heidari, H. Saghaei, F. Koohi-Kamali, M. Naser Moghadasi, en M. K. Moravvej-Farshi, "Proposal for Supercontinuum Generation by Optofluidic Infiltrated Photonic Crystal Fibers," IEEE J. Sel. Top. Quantum Electron. 20(5), (2014).

53. R. Raei, M. Ebnali-Heidari, en H. Saghaei, "Supercontinuum generation in organic liquid-liquid corecladding photonic crystal fiber in visible and near-infrared regions: publisher's note," J. Opt. Soc. Am. B 35(7), 1545 (2018).

54. H. Saghaei en V. Van, "Broadband mid-infrared supercontinuum generation in dispersion-engineered silicon-on-insulator waveguide," J. Opt. Soc. Am. B 36(2), A193 (2019).

55. H. Saghaei en A. Ghanbari, "White light generation using photonic crystal fiber with sub-micron circular lattice," J. Electr. Eng. 68(4), 282-289 (2017).

56. A. Ghanbari, A. Kashani Nia, A. Sadr, en H. Saghaei, "A Comparative Study of Multipole and Empirical Relations Methods for Effective Index and Dispersion Calculations of Silica-Based Photonic Crystal Fibers," J. Commun. Eng. 8(1), 98-109 (2019).

57. M. Kalantari, A. Karimkhani, en H. Saghaei, "Ultra-Wide mid-IR supercontinuum generation in As2S3 photonic crystal fiber by rods filling technique," Optik (Stuttg). 158, 142-151 (2018).

58. M. Ebnali-Heidari, F. Dehghan, H. Saghaei, F. Koohi-Kamali, en M. K. Moravvej-Farshi, "Dispersion engineering of photonic crystal fibers by means of fluidic infiltration," J. Mod. Opt. 59(16), 1384-1390 (2012).

59. F. Mehdizadeh, H. Alipour-Banaei, en S. Serajmohammadi, "Study the role of non-linear resonant cavities in photonic crystal-based decoder switches," J. Mod. Opt. 64(13), 1233-1239 (2017).

60. Z. Chen, Z. Li, en B. Li, "A 2-to-4 decoder switch in SiGe/Si multimode inteference," Opt. Express 14(7), 2671 (2006). 
61. H. Alipour-Banaei, F. Mehdizadeh, S. Serajmohammadi, en M. Hassangholizadeh-Kashtiban, "A 2* 4 all optical decoder switch based on photonic crystal ring resonators," J. Mod. Opt. 62(6), 430-434 (2015).

62. M. Danaie en H. Kaatuzian, "Bandwidth Improvement for a Photonic Crystal Optical Y-splitter," J. Opt. Soc. Korea 15(3), 283-288 (2011).

63. L. Gu, W. Jiang, X. Chen, L. Wang, en R. T. Chen, "High speed silicon photonic crystal waveguide modulator for low voltage operation," Appl. Phys. Lett. 90(7), 71105 (2007).

64. H. Saghaei, P. Elyasi, en R. Karimzadeh, "Design, fabrication, and characterization of Mach-Zehnder interferometers," Photonics Nanostructures - Fundam. Appl. 37, 100733 (2019).

65. E. Danaee, A. Geravand, en M. Danaie, "Wide-band low cross-talk photonic crystal waveguide intersections using self-collimation phenomenon," Opt. Commun. 431, 216-228 (2019).

66. D. G. S. Rao, V. Palacharla, S. Swarnakar, en S. Kumar, "Design of all-optical D flip-flop using photonic crystal waveguides for optical computing and networking," Appl. Opt. 59(23), 7139-7143 (2020).

67. S. S. Zamanian-Dehkordi, M. Soroosh, en G. Akbarizadeh, "An ultra-fast all-optical RS flip-flop based on nonlinear photonic crystal structures," Opt. Rev. 25(4), 523-531 (2018).

68. A. Poustie, R. J. Manning, A. E. Kelly, en K. J. Blow, "All-optical binary counter," Opt. Express 6(3), 69 (2000).

69. S. Kaur en R. S. Kaler, "5 GHz all-optical binary counter employing SOA-MZIs and an optical NOT gate," J. Opt. (United Kingdom) 16(3), 35201 (2014).

70. K. Wen, Y. Hu, L. Chen, J. Zhou, L. Lei, en Z. Guo, "Fano resonance with ultra-high figure of merits based on plasmonic metal-insulator-metal waveguide," Plasmonics 10(1), 27-32 (2015).

71. B. Luk'yanchuk, N. I. Zheludev, S. A. Maier, N. J. Halas, P. Nordlander, H. Giessen, en C. T. Chong, "The Fano resonance in plasmonic nanostructures and metamaterials," Nat. Mater. 9(9), 707-715 (2010).

72. J. Butet en O. J. F. Martin, "Fano resonances in the nonlinear optical response of coupled plasmonic nanostructures," Opt. Express 22(24), 29693-29707 (2014).

73. V. V Klimov, A. A. Pavlov, I. V Treshin, en I. V Zabkov, "Fano resonances in a photonic crystal covered with a perforated gold film and its application to bio-sensing," J. Phys. D. Appl. Phys. 50(28), 285101 (2017).

74. F. Peng, Z. Wang, G. Yuan, L. Guan, en Z. Peng, "High-sensitivity refractive index sensing based on Fano resonances in a photonic crystal cavity-coupled microring resonator," IEEE Photonics J. 10(2), 1-8 (2018).

75. N. Liu, T. Weiss, M. Mesch, L. Langguth, U. Eigenthaler, M. Hirscher, C. Sonnichsen, en H. Giessen, "Planar metamaterial analogue of electromagnetically induced transparency for plasmonic sensing," Nano Lett. 10(4), 1103-1107 (2010).

76. G. U. O. Yuan, X. U. Xue-mei, Y. I. N. Lin-zi, D. Yi-peng, en D. Li-rong, "Study on the Transmission Characteristics of the Fano Resonance Based on the Semi-ring Structure," Acta Photonica Sin. 47(1), 124002 (2018).

77. J. Zhou, H. Chen, Z. Zhang, J. Tang, J. Cui, C. Xue, en S. Yan, "Transmission and refractive index sensing based on Fano resonance in MIM waveguide-coupled trapezoid cavity," AIP Adv. 7(1), 15020 (2017).

78. R. Zafar, S. Nawaz, G. Singh, A. d'Alessandro, en M. Salim, "Plasmonics-based refractive index sensor for detection of hemoglobin concentration," IEEE Sens. J. 18(11), 4372-4377 (2018).

79. M. Wang, M. Zhang, Y. Wang, R. Zhao, en S. Yan, "Fano resonance in an asymmetric MIM waveguide structure and its application in a refractive index nanosensor," Sensors 19(4), 791 (2019). 


\section{Figures}

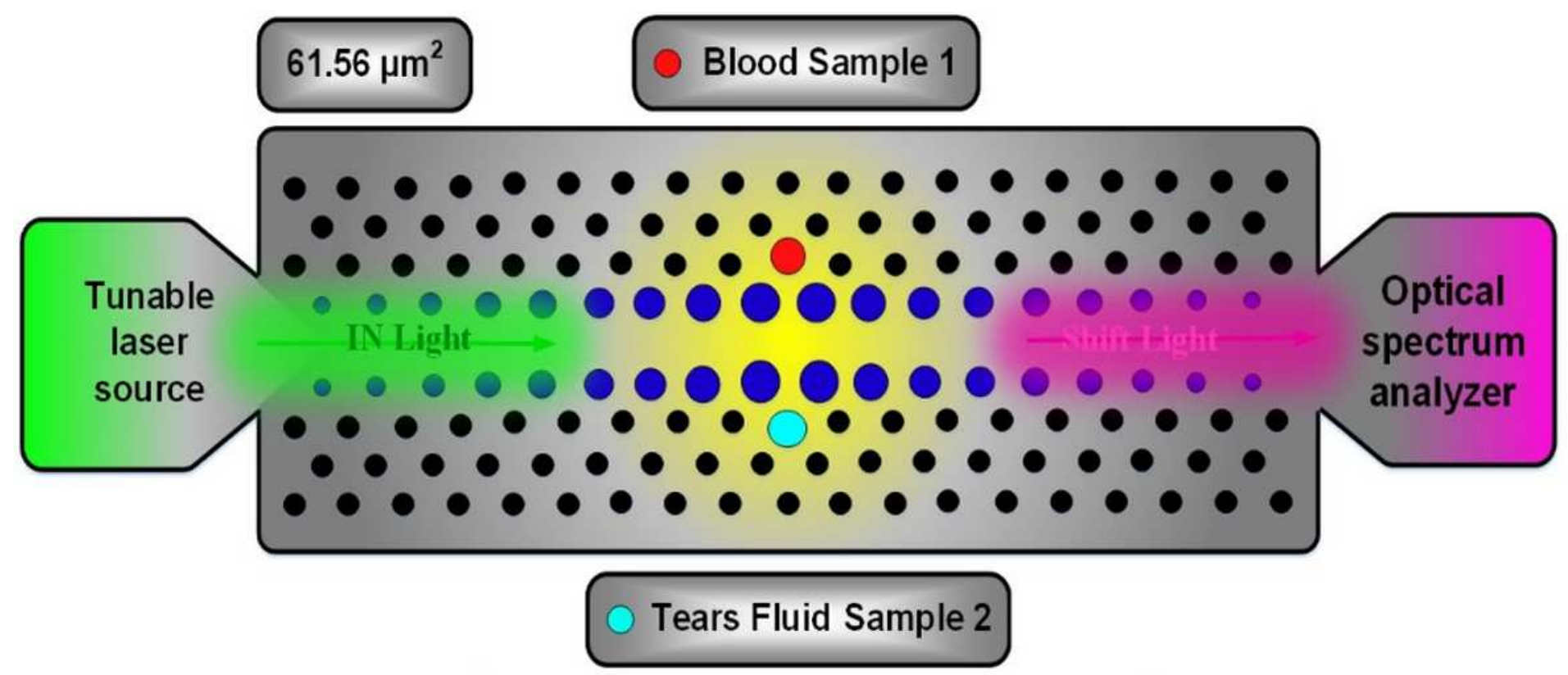

Figure 1

The proposed structure of PhC-based biosensor.
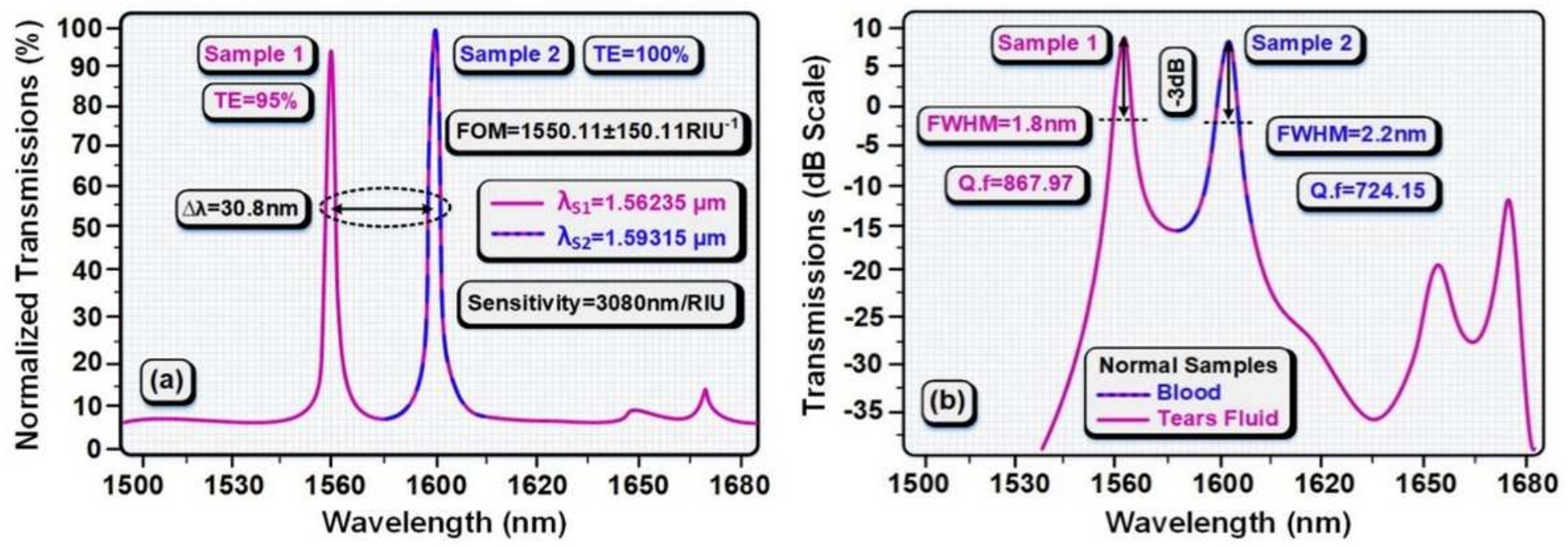

Figure 2

Resonance wavelengths of normal blood and tear samples, (a) Normalized resonance power, (b) Normalized resonance power in $\mathrm{dB}$ scale. 

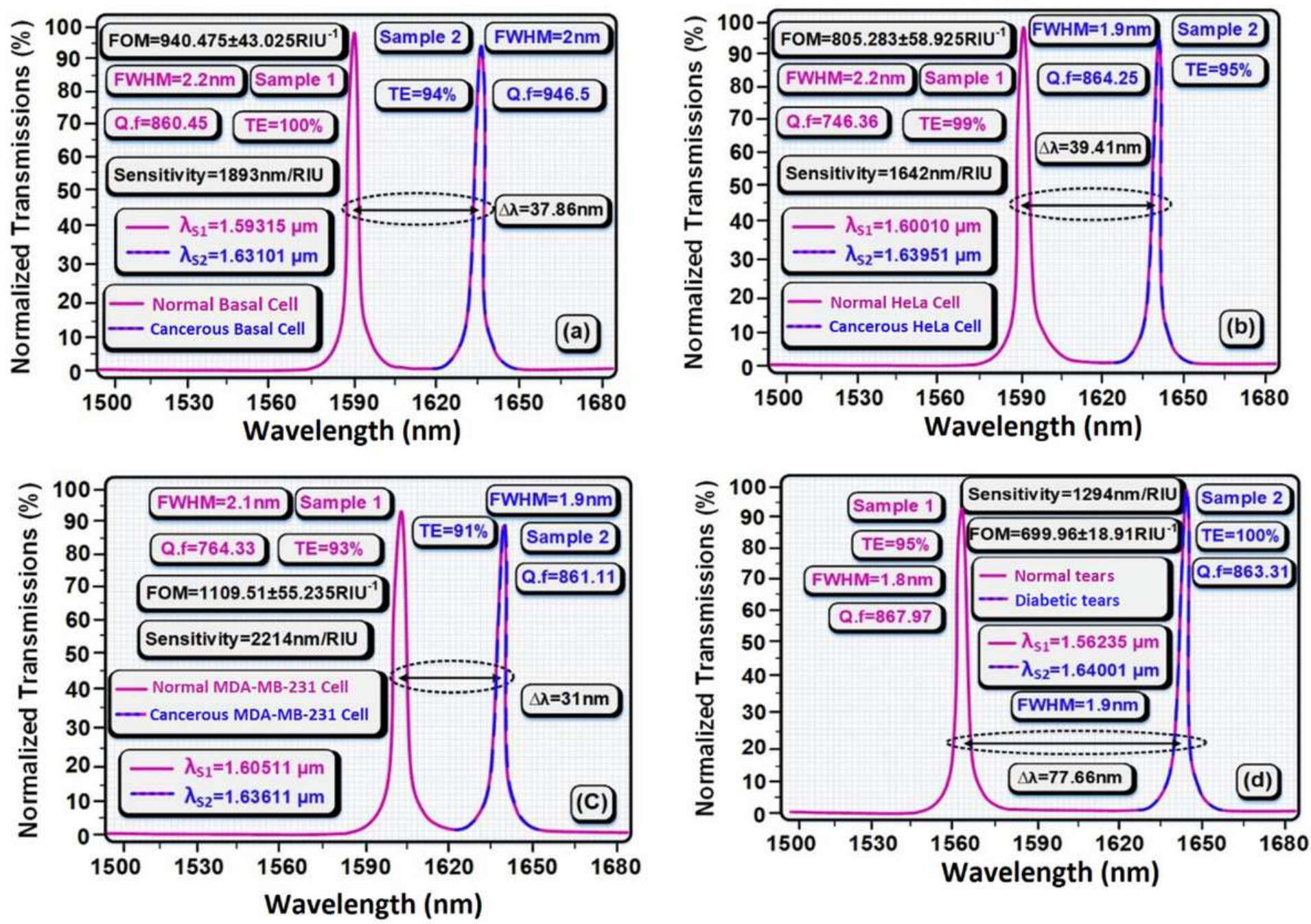

Figure 3

Resonant wavelengths of normal and cancerous cells for (a) Basal cell, (b) HeLa cell, and (c) MDA-MB231 cell and also (d) normal and diabetic tears.
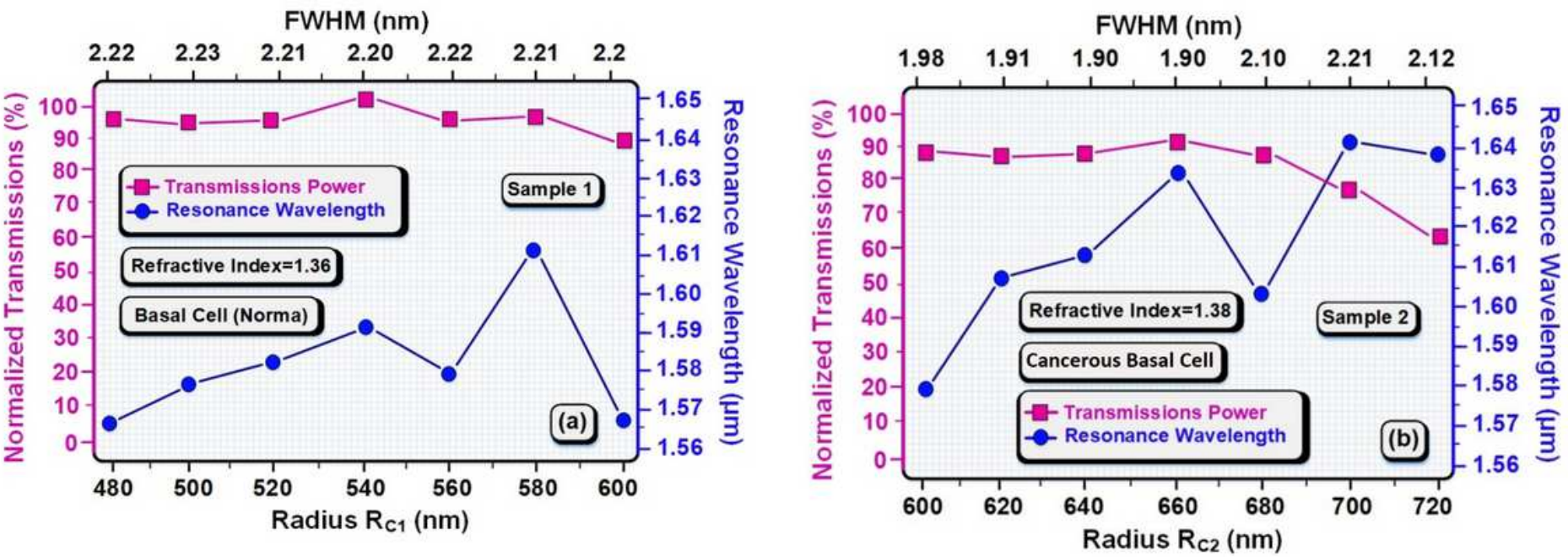

Figure 4 
The resonant wavelengths, transmission power, and FWHM for different tube radius. (a) Normal cell. (b) Cancer cell. 